\title{
NOUVELL
}

\section{Dépistage du cancer colorectal : un test fiable fondé sur la détection des mutations d'APC}

Hélène Blons

$>$ Le développement de tests spécifiques et sensibles permettant un diagnostic précoce de la maladie cancéreuse est un enjeu important d'un point de vue clinique et économique car il doit permettre une meilleure prise en charge des malades à un moindre coût. Une politique de dépistage est particulièrement nécessaire dans le cas du cancer du côlon qui représente la deuxième cause de mortalité par cancer - la première chez les non fumeurs - et pour lequel une prise en charge précoce est un facteur de survie majeur. Par ailleurs, l'incidence du cancer du côlon en France justifie un dépistage de masse qui ne peut se concevoir que dans le respect de certaines conditions, de sensibilité, spécificité et faisabilité pour le test, de sécurité pour le malade. Les outils de dépistage conventionnels sigmoïdoscopie, radiographie et coloscopie - ne répondent à ces objectifs ni en termes de coût ni en termes de risque. Les tests de détection du sang dans les selles, dont la présence traduit l'existence d'ulcérations et la fragilité épithéliale des tumeurs, présentait un avantage important en termes de faisabilité et de sécurité. Aujourd'hui, l'Hémoccult ${ }^{\circledR}$ est admis en dépistage de masse et, malgré une sensibilité très moyenne, notamment dans le cas du diagnostic des adénomes de haut grade, ce test permet un gain en terme de mortalité cumulée [1, 2], mais la proportion de 5 à $10 \%$ de faux positifs contribue à augmenter de façon importante les coûts des programmes de dépistage. L'amélioration de la spécificité de ces tests n'est possible qu'au prix d'une diminution de la sensibilité. Or, un manque de spécificité et de sensibilité discrédite les analyses auprès du public et rend très difficile la conduite d'une politique efficace de dépistage. En outre, sa réalisation nécessite des prélèvements itératifs et un régime alimentaire excluant les viandes rouges et la prise d'anti-inflammatoires non-stéroïdiens, ce qui représente une entrave à la compliance des sujets. De ce fait, la recherche et le développement de nouveaux outils diagnostiques du cancer colorectal reste un sujet d'actualité.

Le développement des techniques de biologie moléculaire, leur possible automatisation pour le traitement de grandes séries d'échantillons, et l'identification de nombreuses altérations moléculaires spécifiques des cellules tumorales coliques ont été à l'origine de la conception de tests fondés sur la détection d'altérations génétiques spécifiques de tumeurs dans les selles des sujets. Ces altérations génétiques, utilisées comme marqueur, témoignent de la présence de cellules tumorales dans la lumière colique. Leur utilisation est néanmoins encore limitée par la nécessité de développer des techniques de biologie moléculaire suffisamment sensibles afin de permettre la mise en évidence d'un génome muté parmi de nombreux génomes normaux. Le marqueur moléculaire idéal serait une altération fréquente, précoce et spécifique qui permettrait de dépister un grand nombre de sujets et de diagnostiquer les stades précancéreux. Des mutations des gènes KRAS, TP53 et APC (adenomatous polyposis coli) ont été caractérisées, montrant la faisabilité de telles études $[3,4]$. Cependant, des mutations de KRAS sont retrouvées dans le cadre de maladies non tumorales et leur utilisation comme marqueur diminue la spécificité du test.

Dans une étude récente, l'équipe de Vogelstein a développé une technique de détection des mutations du gène $A P C$ dans les selles de malades [5]. Vingt-huit malades atteints d'un cancer colorectal non métastatique, 18 malades avec un adénome de plus de $1 \mathrm{~cm}$ et 28 témoins ayant une coloscopie normale ont été inclus dans cette étude. Les selles ont été recueillies avant la coloscopie. L'ADN a été extrait à partir de $4 \mathrm{~g}$ de selles. Un point chaud de mutation dans les néoplasies colorectales est localisé entre les codons 1250 et 1500 du gène $A P C$, regroupant plus de $80 \%$ des mutations somatiques du gène $A P C$. Cette région est située au niveau de l'exon 15 du gène $A P C$ qui regroupe plus de la moitié de la séquence codante $d u$ gène. La très grande majorité des mutations du gène $A P C$ sont des mutations stop. II en résulte la synthèse d'une protéine tronquée, ce qui permet l'identification des mutations après traduction in vitro. En effet, la présence d'un produit plus court par rapport à la protéine attendue signe la présence d'une mutation du gène $A P C$.

L'ADN extrait des selles a été enrichi en cibles APC par hybridation avec des séquences complémentaires biotinylées spécifiques du gène $A P C$. Pour chaque échantillon, 144 PCR ont été effectuées sur l'extrait enrichi en utilisant de 1 à 4 équivalent génomes. Ainsi, un petit nombre de fragments d'ADN APC sont introduits dans chaque $P C R$, ce qui évite la dilution des fragments correspondant à 
I'ADN muté par ceux qui correspondent au gène $A P C$ non muté provenant de l'exfoliation de cellules saines. Cela explique pourquoi, pour obtenir une bonne sensibilité, I'analyse doit être répétée 144 fois. Les produits de PCR ont ensuite été soumis à une traduction in vitro. Un test a été considéré comme positif si au moins 2 des 144 PCR montraient un produit de plus petite taille. La sensibilité de la méthode de détection des mutations APC a été évaluée à une copie mutée pour 250 copies normales.

Les résultats montrent que le test restait négatif chez les 28 sujets témoins, mais était positif chez $61 \%$ des patients atteints de cancer colorectal (IC95\% : 41 - 79\%), et $50 \%$ de ceux qui étaient porteurs d'adénomes de plus de $1 \mathrm{~cm}$ (IC95\% : 26\% - 74\%). Le nombre de copies mutées d'APC était compris entre 0,4 et $14 \%$ de l'ensemble des copies du gène $A P C$ extrait des échantillons de selles. La sensibilité de la méthode de détection des adénocarcinomes et des adénomes de plus de $1 \mathrm{~cm}$ est de $57 \%$ et sa spécificité de $100 \%$.

Cette étude montre que le dépistage des altérations génétiques dans les selles de malades atteints cancers coliques est envisageable et ouvre la voie à la réali- sation de tests moléculaires plus performants que le test Hémoccult ${ }^{\circledR}$. Ils devront être validés dans le cadre d'une politique de dépistage. $\diamond$

Mutations of the $A P C$ gene : reliable markers for an early detection of colic cancer

\section{RÉFÉRENCES}

1. Hardcastle JD, Chamberlain J0, Robinson MH, et al. Randomised controlled trial of faecal-occultblood screening for colorectal cancer. Lancet 1996 ; $348: 1472-7$

2. Mandel JS, Bond JH, Church TR, et al. Reducing mortality from colorectal cancer by screening for fecal occult blood. Minnesota Colon Cancer Control Study. N Engl J Med 1993 ; 328 : 1365-71.

3. Sidransky D, Tokino T, Hamilton SR, et al.
Identification of ras oncogene mutations in the stool of patients with curable colorectal tumors. Science $1992 ; 256$ : 102-5.

4. Ahlquist DA, Skoletsky JE, Boynton KA, et al.

Colorectal cancer screening by detection of altered human DNA in stool: feasibility of a multitarget assay panel. Gastroenterology 2000 ; 119 : 1219-27.

5. Traverso G, Shuber A, Levin $B$, et al. Detection of APC mutations in fecal DNA from patients with colorectal tumors. $N$ Engl J Med 2002 ; 346 : 311-20.

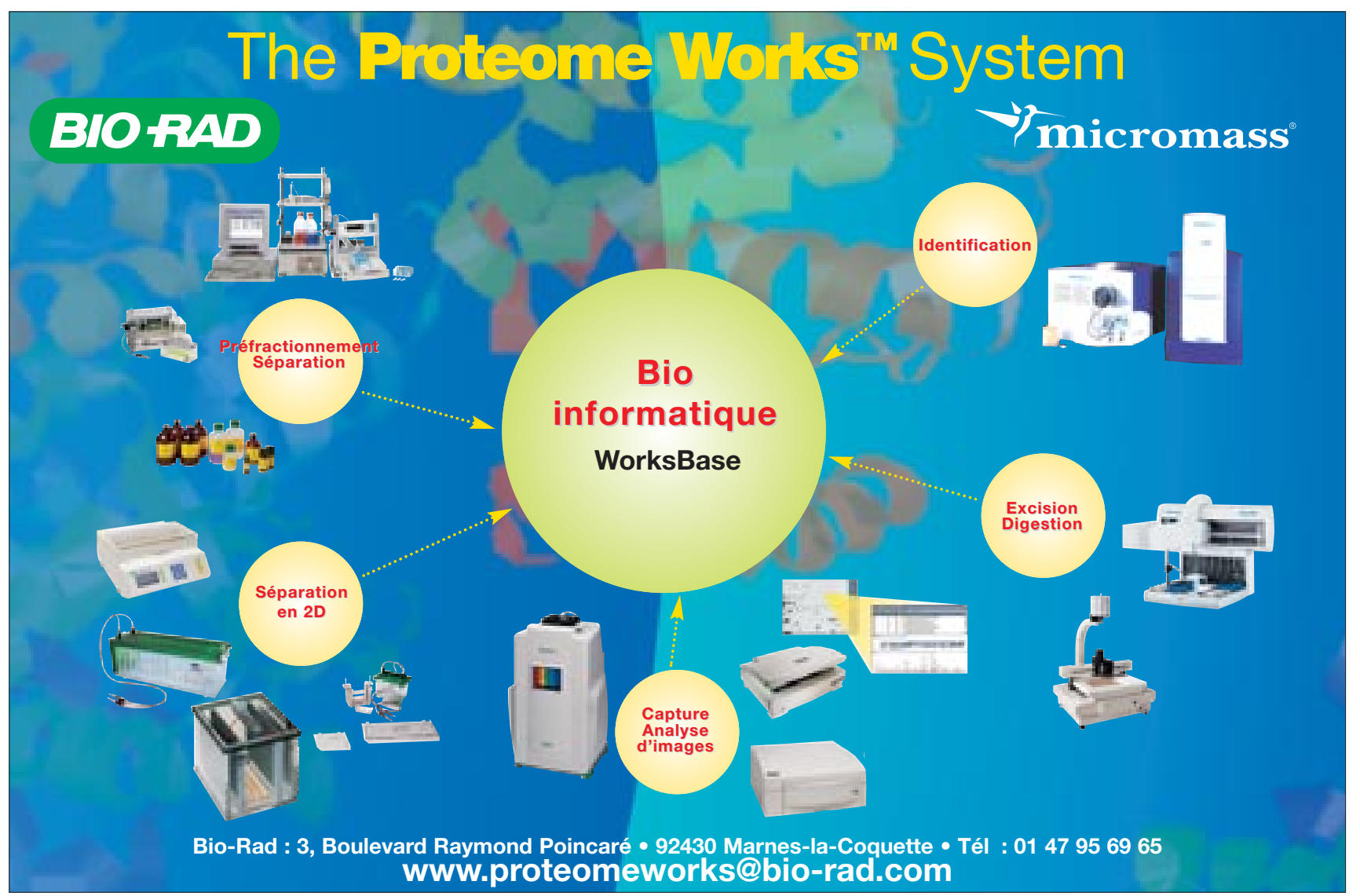

